\title{
A Systematic Study of Extremely Metal-Poor Stars with SDSS/Subaru
}

\author{
Wako Aoki* \\ National Astronomical Observatory of Japan, 2-21-1 Osawa, Mitaka, Tokyo 181-8588, Japan \\ E-mail: aoki.wako@nao.ac.jp
}

\section{Timothy C. Beers}

Department of Physics \& Astronomy and JINA: Joint Institute for Nuclear Astrophysics, Michigan State University, East Lansing, MI 48824-1116 E-mail: beers@pa.msu . edu

\section{Satoshi Honda \\ Kwasan Observatory, Kyoto University, Ohmine-cho Kita Kazan, Yamashina-ku, Kyoto, 607-847, Japan E-mail: honda@kwasan.kyoto-u.ac.jp}

\section{Daniela Carollo}

Research School of Astronomy \& Astrophysics, Australian National University \& Mount Stromlo Observatory, Cotter Road, Weston, ACT, 2611, Australia

E-mail: carollo@mso.anu.edu.au

\section{Hiroko Ito}

The Graduate University for Advanced Studies, 2-21-1 Osawa, Mitaka, Tokyo 181-8588, Japan E-mail: hiroko.ito@nao.ac.jp

\section{Young Sun Lee}

Department of Physics \& Astronomy and JINA: Joint Institute for Nuclear Astrophysics, Michigan State University, East Lansing, MI 48824-1116 E-mail: leeyou25@msu .edu

We have conducted follow-up high-resolution spectroscopy with the Subaru Telescope High Dispersion Spectrograph (HDS) of extremely metal-poor (EMP) star candidates discovered by the Sloan Digital Sky Survey (SDSS), in particular the sub-survey SEGUE, in order to determine accurate metallicities and elemental abundance ratios. Here we report first results for $115 \mathrm{ob}-$ jects, among which 70 stars have $[\mathrm{Fe} / \mathrm{H}]<-3$, doubling the known samples of such stars. We clearly confirm the rapid decrease of the number of stars below $[\mathrm{Fe} / \mathrm{H}]=-3.5$, although the lowmetallicity cutoff is not as sharp as that found by previous studies based on medium-resolution spectra. We identified two cool $(5000 \mathrm{~K})$ main-sequence stars with $[\mathrm{Fe} / \mathrm{H}]<-3$, the first example of this class of stars studied based on high-resolution spectra. One of them exhibits an extremely large enhancement of r-process elements (e.g., $[\mathrm{Eu} / \mathrm{Fe}]=+1.9$ ). The metallicity of this object $([\mathrm{Fe} / \mathrm{H}]=-3.4)$ is the lowest yet found for such r-process-enhanced stars.

11th Symposium on Nuclei in the Cosmos, NIC XI

July 19-23, 2010

Heidelberg, Germany

\footnotetext{
* Speaker.
} 


\section{Extremely Metal-Poor stars and their implications}

Chemical abundance studies of extremely metal-poor (EMP) stars $([\mathrm{Fe} / \mathrm{H}]<-3)$ in the Galactic halo systems provide unique constraints on the nucleosynthesis of first generations of stars and early enrichment in the Galactic halo [1]. We can also obtain constraints on low-mass star formation rate at low metallicity by examination of the statistics of these stars. In addition, the nature of EMP stars may also be closely related to the formation of the proposed components of the Milky Way halo. Although the elemental abundance patterns of the lowest metallicity stars, in particular the three stars with $[\mathrm{Fe} / \mathrm{H}]<-4$ (e.g. Christlieb et al. [2]), have been extensively studied, systematic examination of much larger samples of EMP stars are required to obtain better insight on the formation of first generations of stars and the early enrichment in the Galaxy.

There remain challenges for carrying out such studies. One limiting factor is that EMP stars are extraordinarily rare in the solar neighborhood, and wide-field, deep spectroscopic surveys are required to identify them in any number. Another difficulty is the weakness of spectral lines in the spectra of EMP stars, which makes high-resolution spectroscopy indispensable to obtain elemental abundance information, including even their overall metallicity.

\section{Search for EMP stars from the SDSS/SEGUE sample}

Stellar surveys conducted as part of the Sloan Digital Sky Survey (SDSS; [3]), in particular the Sloan Extension for Galactic Exploration and Understanding (SEGUE; [4]) have provided large samples of candidate EMP stars. The SDSS survey is based on medium-resolution $(R=2000)$ spectra covering the optical range, including the CaII $\mathrm{K}$ line, which enable reasonably accurate estimates of stellar metallicity.

Over the course of a two-year Intensive Program, we obtained high-resolution spectroscopy for candidate EMP stars identified by SDSS using the Subaru Telescope High Dispersion Spectrograph (HDS). We first obtained spectra for some 150 stars with resolving power $R=30,000$, sufficient for resolving weak atomic absorption features in metal-poor stars. Although the $\mathrm{S} / \mathrm{N}$ ratios of these "snapshot" spectra are not very high (25-30), they are adequate for determination of the overall metallicity and abundance ratios of a number of important elements.

While the metallicity determination from SDSS spectra is calibrated by previously well-studied objects in the halo and clusters $[5,6]$, the calibration available at the time our spectra were taken was insufficient for the lowest metallicity range $([\mathrm{Fe} / \mathrm{H}]<-2.5)$. We selected targets with $[\mathrm{Fe} / \mathrm{H}]$ $<-2.6$ from medium-resolution spectra, and found that the iron abundances of most objects determined at high resolution are lower by $0.2-0.3 \mathrm{dex}$; thus, EMP stars are very efficiently selected from the SDSS spectra. However, the correlation between the two estimates is not optimal in this metallicity range, indicating that high-resolution spectroscopy is required to obtain accurate metallicities for individual objects.

It should be noted that the estimates from SDSS, from which our targets were selected, were those obtained prior to 2008. The metallicity estimated by the updated SEGUE Stellar Parameter Pipeline (SSPP; [7]) is expected to be much improved. 


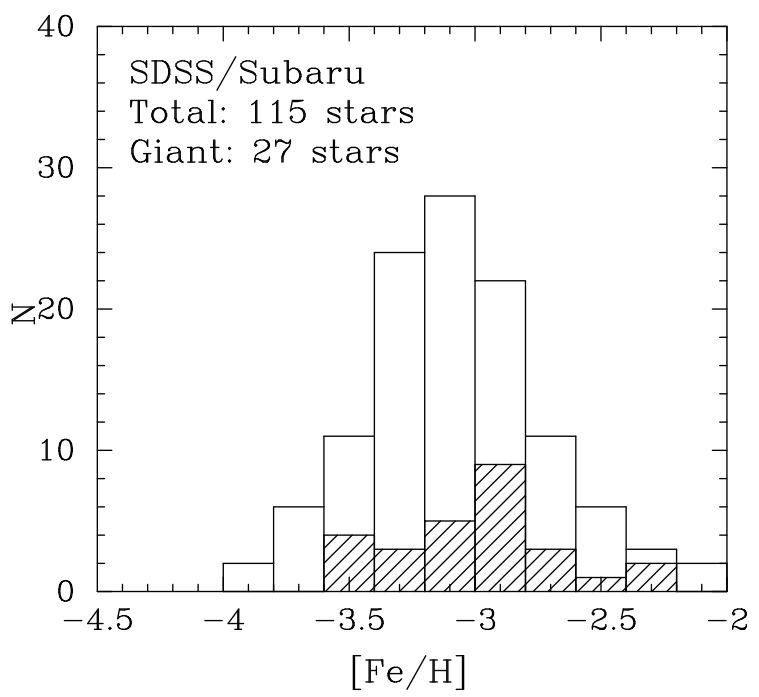

Figure 1: Metallicity distribution obtained from high resolution spectra for our sample. The hatched bins indicate the distribution of red giants, while the others are main-sequence turnoff or subgiant stars. The range of $[\mathrm{Fe} / \mathrm{H}] \gtrsim-3$ is affected by (intentional) selection bias (see text).

\section{Metallicity distribution}

The metallicity distribution function (MDF) of metal-poor stars in the Galactic halo system has been mostly studied based on low- or medium-resolution spectroscopy. Recently, results from the Hamburg/ESO survey (HES) and its follow-up medium-resolution spectroscopy have been reported for main-sequence turnoff stars and red giants separately [8, 9]. Since the HES samples are affected by selection bias in higher metallicity ranges, their discussion focusses on the observed MDF in the very metal-poor range $([\mathrm{Fe} / \mathrm{H}]<-2.0)$, in particular the sharp drop at $[\mathrm{Fe} / \mathrm{H}]$ below -3.5 .

The low-metallicity tail or cutoff of the halo MDF contains important information on the metallicity at which low-mass star formation in the smaller systems (mini halos) that apparently were assembled into the halo took place, as well as on their merging history. Schörck et al. [8] and $\mathrm{Li}$ et al. [9] argued that the sharp cutoff of the MDF at $[\mathrm{Fe} / \mathrm{H}]=-3.5$ found in their sample can only be explained by models that assume some critical metallicity for low-mass star formation. However, we should recall the difficulty in obtaining metallicity estimates from mediumresolution spectroscopy for such extreme stars. Thus, we have considered the MDF derived from high-resolution spectroscopy for our sample.

Figure 1 shows the metallicity distribution obtained for our sample (total of 115 stars) based on high-resolution spectra. From comparisons with the metallicity estimates from SDSS, the range of $[\mathrm{Fe} / \mathrm{H}]>-3$ of our sample certainly reflects a strong selection bias, although it appears mostly free from other biases. For instance, the distribution of visual magnitudes of our sample shows no correlation with metallicity, because we have not explicitly included the most metal-poor candidates in the sample selection. There is also no clear correlation between the metallicity and stellar type (red giants or turnoff stars) in our sample.

Comparison of our result with the HES sample based on medium-resolution spectra, by nor- 


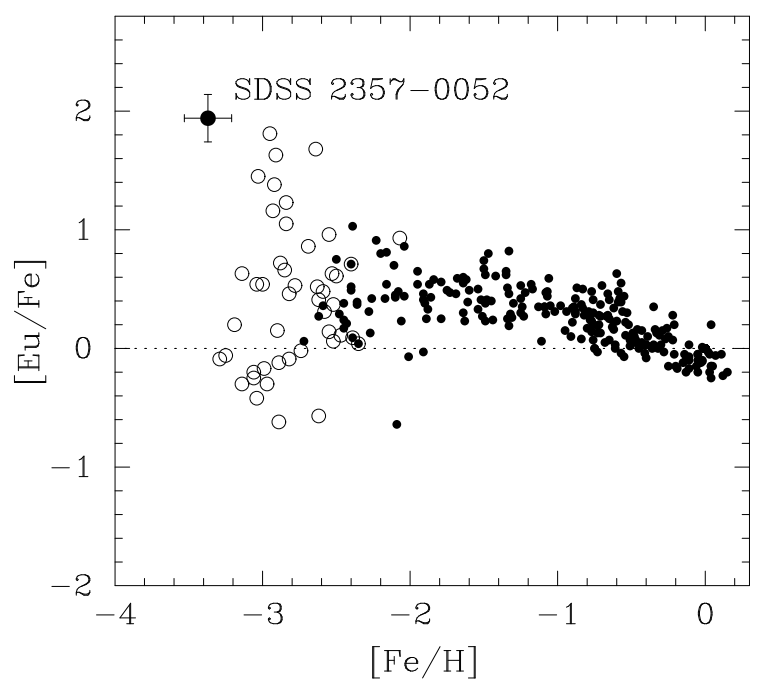

Figure 2: Eu abundance ratios of Galactic field stars. The large filled circle indicates our result for SDSS J2357-0052, while open circles are for very metal-poor stars taken from literature (see Aoki et al. [11]). Results for less metal-poor stars shown by the small filled circles are taken from the SAGA database [13].

malizing the distributions at $[\mathrm{Fe} / \mathrm{H}]=-3$, indicates a significant excess of more metal-poor stars in the SDSS sample. However, this might be due to sensitivity to the normalization cut. For example, if the normalization is made at $[\mathrm{Fe} / \mathrm{H}]=-3.2$, the MDFs in the range $-3.6<[\mathrm{Fe} / \mathrm{H}]<-3.2$ agree very well. Another possible reason for the discrepancy could be systematic errors in the metallicity estimate from high-resolution spectra. If we assume an 0.15 dex offset in our metallicity estimate, which is possible if we adopt different effective temperature scales, the agreement between the two MDF improves a great deal.

However, even taking these difficulties into account, our sample includes several objects in the metallicity range $[\mathrm{Fe} / \mathrm{H}]$ below -3.5 , indicating that the drop in the MDF is not as sharp as found in the HES sample[8, 9]. This may affect interpretations of the MDF at the lowest metallicity.

\section{SDSS J2357-0052: a cool main-sequence EMP star with large r-process excesses}

Among the SDSS sample observed with Subaru, we have identified several cool stars that have significantly higher gravity than red giants. Higher S/N spectra were obtained by followup observations for two such stars. These stars turned out to be cool (effective temperatures of $\sim 5000 \mathrm{~K})$ main-sequence stars with $\log g \sim 4.8$. Previously known EMP stars are mostly red giants, and only a small fraction of main-sequence turnoff stars are known in this abundance range. No cool main-sequence star has been found in previous samples of EMP stars, probably because these stars are intrinsically very faint. An exception is the dwarf carbon star G 77-61 [10]. The two stars in our study are the first examples of cool main-sequence EMP stars of normal carbon abundance with available high-resolution spectroscopy.

A surprising result is that one of the two stars exhibits very large enhancements of heavy neutron-capture elements. This star has relatively weak Fe absorption lines, but clearly shows strong Eu absorption features. The derived $[\mathrm{Fe} / \mathrm{H}]$ is -3.4 , and $[\mathrm{Eu} / \mathrm{Fe}]$ is +1.9 . The abundance 
pattern of seven elements ( $\mathrm{Sr}, \mathrm{Y}, \mathrm{Ba}, \mathrm{La}, \mathrm{Eu}, \mathrm{Er}$ and $\mathrm{Yb}$ ) agrees well with the r-process abundance pattern estimated from solar-system material [11].

Figure 2 shows $[\mathrm{Eu} / \mathrm{Fe}]$ ratios as a function of metallicity. Our new r-process-enhanced star SDSS J2357-0052 has the lowest $[\mathrm{Fe} / \mathrm{H}]$, as well as the highest $[\mathrm{Eu} / \mathrm{Fe}]$, among the r-processenhanced stars known to date. Previous studies found r-process-enhanced $([\mathrm{Eu} / \mathrm{Fe}]>+1)$ stars only at $[\mathrm{Fe} / \mathrm{H}] \sim-3$, or slightly higher, metallicity. Our discovery of SDSS J2357-0052 extends the metallicity range for Eu enhancement to $[\mathrm{Fe} / \mathrm{H}]=-3.4$.

The metallicity range in which highly r-process-enhanced stars are found provides a constraint on the astrophysical sites of the r-process. One possible explanation for a limited range is that the progenitors of supernovae that are associated with the r-process are less massive stars $\left(8-10 \mathrm{M}_{\odot}\right)$, which might contribute only to the range of $[\mathrm{Fe} / \mathrm{H}] \gtrsim-3$ (e.g., [12]) . Our discovery suggests that a larger range of progenitor masses may have been involved. Also, there seems to exist an upper bound of r-process-excesses of $[\mathrm{Eu} / \mathrm{H}] \sim-1.5([\mathrm{Eu} / \mathrm{Fe}]=+1.5$ at $[\mathrm{Fe} / \mathrm{H}]=-3.0)$. If typical yields of Eu by a single r-process event are assumed (e.g., Wana jo \& Ishimaru [12]), a limit in the pollution of interstellar matter by a single r-process event can be derived [11]. Greatly expanded samples of r-process-enhanced stars are obviously required to derive definitive conclusions.

\section{References}

[1] A. Frebel, this volume (2010)

[2] N. Christlieb, et al., A stellar relic from the early Milky Way, Nature 419 (904) 2002

[3] D. G. York, et al., The Sloan Digital Sky Survey: Technical Summary, AJ, 120, (1579) 2000

[4] B. Yanny, et al., SEGUE: A Spectroscopic Survey of 240,000 Stars with $g=14-20$, AJ 137 (4377) 2009

[5] Y. S. Lee, et al., The SEGUE Stellar Parameter Pipeline. I. Description and Comparison of Individual Methods, AJ, 136 (2022) 2008

[6] C. Allende Prieto, et al. The SEGUE Stellar Parameter Pipeline. III. Comparison with High-Resolution Spectroscopy of SDSS/SEGUE Field Stars, AJ 136 (2070) 2008

[7] J. P. Smolinski, et al., The SEGUE Stellar Parameter Pipeline. IV. Validation with an Extended Sample of Galactic Globular and Open Clusters, AJ, in press (ArXiv: 1008.1959)

[8] T. Schörck et al., The stellar content of the Hamburg/ESO survey. V. The metallicity distribution function of the Galactic halo, A\&A $\mathbf{5 0 7}$ (817) 2009

[9] H. N. Li, et al. 2010, The stellar content of the Hamburg/ESO survey VI. The metallicity distribution of main-sequence turnoff stars in the Galactic halo, A\&A, $\mathbf{5 2 1}$ (10) 2010

[10] B. Plez, \& J. G. Cohen, Analysis of the carbon-rich very metal-poor dwarf G77-61, A\&A 434 (1117) 2005

[11] Aoki, W. et al. Extreme Enhancements of r-process Elements in the Cool Metal-Poor Main-Sequence Star SDSS J2357-0052, ApJL $\mathbf{7 2 3}$ (L201) 2010

[12] S. Wanajo, Y. Ishimaru, r-process calculations and Galactic chemical evolution, Nuclear Physics A 777 (676) 2006

[13] T. Suda et al., Stellar Abundances for the Galactic Archeology (SAGA) Database - Compilation of the Characteristics of Known Extremely Metal-Poor Stars, PASJ 60 (1159) 2008 